\title{
Analysis of the Supervision of Ecological Subsidies: Based on the Principal-agent Model
}

\author{
Yuesheng Zhang ${ }^{1,2^{+}}$ \\ ${ }^{1}$ School of Finance and Economics, Xi'an Jiaotong University, Shaanxi 710061, China \\ ${ }^{2}$ XinXiang University, Henan 453003, China
}

\begin{abstract}
In view of the problem of the invalidity of the incentive mechanism of the ecological subsidies, which is due to the information asymmetry between the government's supervision and the enterprise endeavor to fulfill their ecological responsibilities, this paper attempts to analyze the supervision of ecological subsidies based on the Principal-agent Model. Two conclusions are drawn: firstly, the government's supervision regarding the effect of the enterprises' fulfilling the ecological responsibilities can significantly reduce the information asymmetry; secondly, the government's incentive strength and the enterprises' endeavor level of fulfilling the ecological responsibilities are both improving the surveillance dynamics. Here is the suggestion: with the increasing of the surveillance dynamics of the government and the transparency of the enterprises' fulfilling the ecological responsibilities, the government should meanwhile increase the subsidies incentive strength, therefore, to promote the effort level of the enterprises' fulfilling the ecological responsibilities to approach to the Pareto optimal value.
\end{abstract}

Keywords: Ecological subsidies, Information asymmetry, Principal-agent model, Supervision

\section{Introduction}

The deterioration of the ecological environment has become the key constraint of the sustainable development of the Chinese economy. Therefore, the issue of ecological subsidy has begun to attract much attention from all sectors of government and society, it also has become a research focus in many fields including ecology, resource, environment and the coordinated regional development. Nowadays, the term 'ecological subsidy' has been widely referred to and taken use of, and frequently appears in the media, the government's working reports and academic researches (e.g., On January 12, 2013, the writer has retrieved 4,030,000 relevant web pages with the key word of 'ecological subsidy' via Baidu (China). And 2,465 articles in Chinese whose titles contain the term 'ecological subsidy' are retrieved via CNKI (China), including 2,143 journal articles, 285 master's theses and 37 doctor's dissertations, 1,660 of which are published after 2009). While we perfecting the ecological subsidy mechanism, it is inevitable to make a research about the supervision of the ecological subsidy. According to Kagan et al. [1], the regulatory supervision of the environmental governance plays an important role in the environmental improvement of the developed countries in the past three decades. Similarly, Doonan et al. [2], Delmas and Toffel [3] all hold that supervision exert an important influence on motivation of the government's performing its responsibility to protect the environment.

Because both the government and the enterprises have their own information gathering channels, they master the information which is unknown to the other. But in general, as a micro entity of the economic operation, the enterprises own more information. While the regulation departments of the government cannot master the real and comprehensive information, therefore they cannot effectively observe the real situation of the enterprises' fulfilling their 'ecological' responsibility. In order to full play the incentive effect of the ecological subsidies, the government has to focus on the supervision of the enterprises' ecological subsidies. Observation and supervision work must be conducted to identify the efforts of the enterprises to fulfill the 'ecological' responsibility so that the ecological subsidies can be well performed and the environment can be well improved. Although the government cannot observe the real behaviors of the enterprises, the other outer factors that influence the efforts of the enterprises to fulfill the 'ecological' responsibility are easier to be observed. The government can estimate the enterprises' efforts by observing and supervising the natural situation of the enterprises' fulfilling the 'ecological' responsibility. Thus the information asymmetry can be reduced to a certain extent. So, this article makes an analysis
cC (P) $\$$ This is an Open Access article distributed under the terms of the Creative Commons Attribution Non-Commercial License (http://creativecommons. org/ licenses/by-nc/3.0/) which permits unrestricted non-commercial use, distribution, and reproduction in any medium, provided the original work is properly cited.
Received September 5, 2104 Accepted December 4, 2014

$\uparrow$ Corresponding author

Email: xxtc2008@163.com

Tel: +86-139-3730-6911 Fax: +86-037-3368-3552

Copyright (C) 2014 Korean Society of Environmental Engineers 
of the government's supervision over the ecological subsidy based on the Principal-agent Model in order to provide some helpful supports for the establishment of the ecological subsidy mechanism in China.

\section{The Principal-agent Supervision Model of the Ecological Subsidy}

\subsection{Fundamental Hypothesis}

- Hypothesis 1: the comprehensive benefits which came from the enterprises' fulfilling their 'ecological' responsibility are directly relevant to the efforts they've made. Assuming the benefits $\pi$ brought about by the enterprises' fulfilling the 'ecological' responsibility is linear with their efforts $\alpha$ :

$$
\pi=k \alpha+\varphi
$$

Wherein, $\alpha$ refers to the efforts of the enterprises' fulfilling their 'ecological' responsibility. It is mainly decided by the resource investment which is mastered by the enterprises and can also be taken as the effective action adopted by the enterprises in accordance with the government's commission. It is the most important variable to reflect the enterprises' efforts to make ecological subsidies, which is beyond the government's observation. $k$ refers to the conversion constant for the enterprises' efforts being converted into benefits. $\varphi$ refers to the exogenous uncertainty variable and it can be called the natural condition.

- Hypothesis 2: the natural condition $\varphi$ subjects to normal distribution, in other words, it subjects to $N\left(\mu, s^{2}\right)$. The government's observation effort of the natural condition $\varphi$ is $\chi$. Observation cost is $w(\chi)$. The expectation value and variance after the government's observation of the natural condition respectively are $x(\chi)$ and $y(\chi)$. Therefore, the expectation value and variance of the output $\pi$ is:

$$
\begin{gathered}
E(\pi)=k \alpha+E(\varphi)=k \alpha+x(\chi) \\
\operatorname{Var}(\pi)=y(\chi)
\end{gathered}
$$

- Hypothesis 3: the government adopts two types of compensation for the enterprises' fulfilling the 'ecological' responsibility, namely, direct compensation and indirect compensation. We assume $s(\pi)$ to be the compensation contract between the government and the enterprises, which can also be taken as the benefits assigned by the government to the enterprises. Assuming it has a linear form as well:

$$
s(\pi)=b+\beta \pi
$$

Where, $b$ presents the government budget compensation provided by the government to the enterprises because of their active performing the 'ecological' responsibility, which is the enterprises' regular income and has nothing to do with $\pi$. $\beta \pi$ refers to the indirect compensation provided by the government to the enterprises because of their active performing the 'ecological' responsibility. $\beta$ refers to the ratio or the incentive intensity of the enterprises' sharing the benefits from the ecological compensation designed by the government. That is to say, the enterprises will obtain a compensation of $\beta$ unit with per unit increasing of the benefits $\pi$ from the enterprises' performing the 'ecological' responsibility.

- Hypothesis 4: when it comes to the risks, this article assumes the government is the risk-neutral entity and the enterprise is the risk aversion. Then, in the formula (4), $0 \leq \beta \leq 1$ and $\beta=0$ means the enterprise does not have to take any risks. $\beta=1$ means the enterprise has to bear all the risks. Based on the hypothesis that the enterprise is the risk aversion, a further hypothesis is made that the enterprise's utility function has a stable feature of risk aversion. Then enterprise's simplified utility function of the risk aversion is $u=-e^{-\rho \tau}$. Wherein, $\rho$ refers to the metric coefficient of the enterprise's absolute risk aversion level (i.e., $\rho$ is put forward by Arrow and Pratt. With the $\rho$ increasing, the party approaches to be risk averse. $\rho<0$ reflects the party is a risk seeker. $\rho=0$ reflects the party is risk neutral. $\rho>0$ reflects the party is risk averse.). $\tau$ refers to the enterprise's actual monetary benefits.

- Hypothesis 5: assuming the enterprise's effort cost $c(\alpha)$ equals to the currency cost. Its effort cost has two important properties, namely, $c^{\prime}(\alpha)>0$ and $c^{\prime \prime}(\alpha)>0$. This demonstrates the more efforts the enterprise makes, the more cost it should pay. And with their increasing efforts, the cost will also raise. To be simplified, assuming the effort cost function of the enterprises is: $c(\alpha)=\frac{1}{2} d \times a^{2}$. Wherein, $d>0$ refers to the cost coefficient. The bigger it is, the more cost under the same effort will cause, or in other words, the larger the negative effects will be.

\subsection{The Setting of the Utility Function}

\subsubsection{The Setting of the Government's Utility Function}

Government's goal of ecological compensation is to urge the enterprises to actively perform their 'ecological' responsibility and try to maximize the social benefits. This utility function refers to the differential between the benefits created by the enterprises' fulfilling the 'ecological' responsibility and the cost caused by the government's ecological compensation. This article sets the government's utility function as:

$$
v[\pi-s(\pi)]=\pi-s(\pi)-w(\chi)
$$

According to the assumption that government is the risk-neutral in the hypothesis 4 , it can be drawn that the government's expectation utility is also its expected return. We can see it in the formula:

$$
\begin{aligned}
& E(v)=E[\pi-b-\beta \pi-w(\chi)] \\
& =-b+(1-\beta)[k \alpha+x(\chi)]-w(\chi)
\end{aligned}
$$

\subsubsection{The Setting of the Enterprise's Utility Function}

The enterprise's motivation to fulfill the 'ecological' responsibility depends on the economic benefits brought about by the activity. But, the ecological compensation will necessarily bring about great 
spillover benefits. Therefore, the enterprise's motivation to fulfill the 'ecological' responsibility mainly depends on the compensation of the spillover effect made by the government. For the convenience of calculation, we assume the enterprise's utility function is the one of the government's ecological compensation as follows:

$$
u[s(\pi)]-c(\alpha)
$$

With consideration of the hypothesis 2 and 4, we can obtain the enterprise's actual monetary income of ecological compensation:

$$
v=s(\pi)-c(\alpha)=b+\beta \pi-\frac{1}{2} d \alpha^{2}=b+\beta(k \alpha+\varphi)-\frac{1}{2} d \alpha^{2}
$$

Therefore, the enterprise's expectation utility should be:

$$
E u=-E\left(e^{-\rho \tau}\right)=-e^{-\rho\left[E \tau-\frac{1}{2} \rho \operatorname{Var}(\tau)\right]}
$$

According to the definition of the certainty equivalent $(C E)$ (i.e., If $x$ presents certainty income variable and $y$ presents random income variable, when $u(x)=E u(y)$, the expectation utility brought about by the random variable $y$ equals to the benefits from the certainty income. Therefore, $x$ can be taken as the certainty equivalent of $y$. When the customers are risk neutral, the certainty equivalent equals to the mean of the random income. When the customers are risk averse, the certainty income equals to the mean of the random income menus the risk cost.), it can be derived that $E u=u(C E)$. So the enterprise's certainty equivalent should be:

$$
C E=E(v)-\frac{1}{2} \rho \beta^{2} \sigma^{2}=b+\beta[k \alpha+x(\chi)]-\frac{1}{2} \rho \beta^{2} y(\chi)-\frac{1}{2} d \alpha^{2}
$$

Wherein, $E(v)$ presents the enterprise's expected return. While $\frac{1}{2} \rho \beta^{2} y(\chi)$ presents the enterprise's risk cost.

On the condition of $\beta=0$, the risk cost equals 0 , which means the enterprise do not have to take any risks, the enterprise do not own the quota of the indirect compensation, and it can only get the regular income. Thus, the maximum expectation utility function of the enterprise is $E u=-E e^{-\rho \tau}$, which equals to the maximum certainty income.

A definite conclusion can be drawn when $\bar{v}$ is the enterprise's revenue reserve that when the certainty income is less than $\bar{v}$, the enterprise will not be willing to accept the existing compensation contract, that's to say, the enterprise doesn't have the motivation to fulfill the 'ecological' responsibility. Therefore, the formula of the constraint condition for the enterprise to take part in should be:

$$
b+\beta[k \alpha+x(\chi)]-\frac{1}{2} \rho \beta^{2} y(\chi)-\frac{1}{2} d \alpha^{2} \geq \bar{v}
$$

\subsection{The Ecological Principal-agent Supervision Model}

Taking a reference to the Principal-agent Model of Mirless [4, 5], Holmstrom [6] and Theilen [7], the model of the government's supervision over the natural condition of the enterprise's fulfilling the 'ecological' responsibility should be:

$$
\begin{gathered}
\operatorname{Max} E(v)=-b+(1-\beta)[k \alpha+x(\chi)]-w(\chi) \\
\text { s.t. (IR) } b+\beta[k \alpha+x(\chi)]-\frac{1}{2} \rho \beta^{2} y(\chi)-\frac{1}{2} d \alpha^{2} \geq \bar{v} \\
\text { (IC) } \alpha \in \arg \operatorname{Max}\left\{b+\beta[k \alpha+x(\chi)]-\frac{1}{2} \rho \beta^{2} y(\chi)-\frac{1}{2} d \alpha^{2}\right\}
\end{gathered}
$$

\section{Results and Discussion}

\subsection{Bayesian Analysis of the Asymmetric Information}

Before solving the supervision model of the ecological compensation, we should figure out whether the government can effectively solve the problem of asymmetric information while supervising the natural condition. It can be achieved with the method of Bayesian Analysis.

A hypothesis is made that the observation variable relevant to the natural condition $\varphi$ that the government can obtain is $i$. According to Bayesian theorem, the posterior probability of $\varphi$ determined by $i$ should be:

$$
f(\varphi \mid i)=\frac{q(i \mid \varphi) f(\varphi)}{w(i)}
$$

$q(i \mid \varphi)$ refers to the occurring probability of the observation variable $i$ when the natural condition is $\varphi, w(i)=\int q(i \mid \varphi) f(\varphi) d \varphi . f(\varphi)$ refers to the density function of the natural condition $\varphi$.

In general, the greater the intensity of the government's observations is, the closer the observation variable $i$ will get to the natural condition $\varphi$. Assuming $i=\chi \varphi+(1-\chi) r$ and $r$ subjects to normal distribution $N\left(0, \sigma^{2}\right), \chi \in[0,1]$. On the condition of $\chi=0$, the government cannot observe the natural condition $\varphi$ at all. On the condition of $\chi=1$, the government can utterly observe the natural condition $\varphi$. Therefore, when the natural condition $\varphi$ given, observation variable $i$ subjects to normal distribution $N\left(\chi \varphi,(1-\chi)^{2} \sigma^{2}\right)$ and taking $q(\chi)$ for the intensity function of the observation variable $i$, we can get:

$$
q(i \mid \varphi)=\frac{1}{\sqrt{2 \pi}(1-\chi) \sigma} \exp \left[-\frac{(i-\chi \varphi)^{2}}{2(1-\chi)^{2} \sigma^{2}}\right]
$$

Because of $f(\varphi)=\frac{1}{\sqrt{2 \pi} s} \exp \left[-\frac{(\varphi-\mu)^{2}}{2 s^{2}}\right]$, therefore, we can get,

$$
q(i \mid \varphi) f(\varphi)=\frac{1}{2 \pi(1-\chi) \sigma s} \exp \left\{-\frac{1}{2}\left[\frac{(i-\chi \varphi)^{2}}{(1-\chi)^{2} \sigma^{2}}+\frac{(\varphi-\mu)^{2}}{s^{2}}\right]\right\}
$$

So, we can get:

$w(i)=\int q(i \mid \varphi) f(\varphi) d \varphi=\frac{1}{\sqrt{2 \pi \eta}(1-\chi) \sigma s} \exp \left[\frac{1}{2} \frac{(\mu \chi-i)^{2}}{\chi^{2} s^{2}+(1-\chi)^{2} \sigma^{2}}\right]$

Wherein, $\eta=\frac{1}{s^{2}}+\frac{\chi^{2}}{(1-\chi)^{2} \sigma^{2}}$. 
Thus, the posterior probability can be obtained:

$$
f(\varphi \mid i)=\frac{q(i \mid \varphi) f(\varphi)}{w(i)}=\left(\frac{\eta}{2 \pi}\right)^{\frac{1}{2}} \exp \left\{-\frac{1}{2} \eta\left[\varphi-\frac{1}{\eta}\left(\frac{\mu}{s^{2}}+\frac{\chi i}{(1-\chi)^{2} \sigma^{2}}\right)\right]^{2}\right\}
$$

According to the above reasoning process, the posterior probability subjects to the normal distribution with the expectation to be $\frac{1}{\eta}\left[\frac{\mu}{s^{2}}+\frac{\chi i}{(1-\chi)^{2} \sigma^{2}}\right]$, the variance to be $\frac{1}{\eta}$. And its expectation and variance can be presented as:

$$
\begin{gathered}
x(\chi)=\frac{1}{\eta}\left[\frac{\mu}{s^{2}}+\frac{\chi i}{(1-\chi)^{2} \sigma^{2}}\right]=\frac{\mu(1-\chi)^{2} \sigma^{2}+\chi i s^{2}}{\chi^{2} s^{2}+(1-\chi)^{2} \sigma^{2}} \\
y(\chi)=\frac{1}{\eta}=\frac{(1-\chi)^{2} \sigma^{2} s^{2}}{(1-\chi)^{2} \sigma^{2}+\chi^{2} s^{2}}
\end{gathered}
$$

In terms of the variance formula of $y(\chi), \frac{y(\chi)}{s^{2}}=\frac{(1-\chi)^{2} \sigma^{2}}{(1-\chi)^{2} \sigma^{2}+\chi^{2} s^{2}} \leq 1$ can be obtained. Thus, as long as $\chi$ is not 0 , we can surely get $y(\chi)<s^{2}$. It means the observation on the natural condition $\varphi$ can lower the variance and strengthen the certainty. That is to say, when the government makes observation on the natural condition $\varphi$ in some way, the information asymmetry between the government and the enterprises will decrease and therefore the variance $\operatorname{Var}(\pi)$ can be reduced.

\subsection{Model Solutions and Correlation Analysis}

In terms of the formula (14), the incentive compatible equivalent form is its first-order condition: $\alpha=k \beta / d$. Taking it into the monitoring model, by solving the simultaneous equations, we can obtain:

$$
\begin{gathered}
\beta=\frac{k^{2}}{k^{2}+d \rho y(\chi)} \\
\alpha=\frac{k^{3}}{k^{2} d+d^{2} \rho y(\chi)}
\end{gathered}
$$

From the formula (22) and (23), the incentive intensity and the endeavor level are both the functions of the observation intensity. This provides a ground to investigate their relationship.

Firstly, in order to investigate the relationship between the incentive intensity and the observation intensity, by differentiating a partial derivative of $\chi$ in the formula (22), we can obtain:

$$
\frac{\partial \beta}{\partial \chi}=\frac{k^{2} d \rho \sigma^{2} s^{4}\left(1-\chi^{2}\right)}{\left[k^{2}+d \rho y(\chi)\right]^{2}\left[\chi+\sigma^{2} s^{2}(1-\chi)^{2}\right]^{2}} \geq 0
$$

From the formula (24), it can be derived that with the observation intensity of the natural condition $\varphi$ increasing, the information asymmetry between the government and the enterprise will decrease and their transparency will increase. Under this condition, the government should increase the incentive intensity of the government's compensation for the enterprise's fulfilling the 'ecological' responsibility, which will urge the enterprise to actively fulfill the 'ecological' responsibility.
Secondly, in order to investigate the relationship between the endeavor level and the observation intensity, by differentiating a partial derivative of $\chi$ in the formula (23), we can obtain:

$$
\frac{\partial \alpha}{\partial \chi}=\frac{k^{3} \rho \sigma^{2} s^{4}\left(1-\chi^{2}\right)}{\left[k^{2}+d \rho y(\chi)\right]^{2}\left[\chi+\sigma^{2} s^{2}(1-\chi)^{2}\right]^{2}} \geq 0
$$

From the formula (25), it can be known that with the observation intensity of the natural condition $\varphi$ increasing, the enterprise's advantage of the information asymmetry will decrease and the possibility for the enterprise to fulfill the 'ecological' responsibility will accordingly decrease. In the mean time, with incentive intensity of the government increasing, the enterprise's enthusiasm of fulfilling the 'ecological' responsibility will increase. The enterprise's optimize choice is to raise the endeavor level.

In addition, we should compare the expectation utilities of the enterprise's fulfilling the 'ecological' responsibility according to whether the government will make observation of the natural condition.

When the government does not observe the natural condition, which means $\chi=0$, the expectation utility of the output $\pi$ should be:

$$
E\left(\pi_{0}\right)=k \alpha+x(0)=\frac{k^{4}}{k^{2} d+d^{2} \rho s^{2}}+\mu
$$

When the government observe the natural condition, which means $\chi \neq 0$, the expectation utility of the output $\pi$ should be:

$$
E\left(\pi_{1}\right)=k \alpha+x(\chi)=\frac{k^{4}}{k^{2} d+d^{2} \rho y(\chi)}+\frac{\mu(1-\chi)^{2} \sigma^{2}+\chi i s^{2}}{\chi^{2} s^{2}+(1-\chi)^{2} \sigma^{2}}
$$

Because the observation variable $i$ subjects to the normal distribution $N\left(\chi \varphi,(1-\chi)^{2} \sigma^{2}\right)$, we can obtain $E(i)=\chi \varphi=\chi \mu$. With $s^{2}>y(\chi)$, we can get:

$$
E_{i}[\Delta E(\pi)]=\frac{k^{4} d^{2} \rho\left[s^{2}-y(\chi)\right]}{\left(k^{2}+d \rho s^{2}\right)\left[k^{2}+d \rho y(\chi)\right]}>0
$$

With an analysis of the formula (28), we can get the expected benefits of $\Delta E(\pi)$ on $i$ are more than 0 , which means the government's observation over the enterprise's natural condition $\varphi$ is beneficial to the expected output of the enterprise's fulfilling the 'ecological' responsibility.

\section{Conclusions}

Due to the information asymmetry, it is very difficult for the government to supervise the enterprise's endeavor level of fulfilling the 'ecological' responsibility effectively. To the contrary, it's easy for the government to supervise the natural condition. Therefore, in order to obtain the information about the enterprise's endeavor level of fulfilling the 'ecological' responsibility, the government can choose to make supervision over the natural condition. And in theory, the effects of the supervision over the natural condition of the enterprise's fulfilling the 'ecological' responsibility are equiv- 
alent to of the one over the real enterprise behavior. Therefore, this article designs an ecological compensation supervision model that the government can choose to observe the natural condition.

The Bayesian analysis shows the supervision over the natural condition can effectively reduce the information asymmetry between the government and the enterprise and increase the transparency. With the solution of the principal-agent model constructed in this article, three conclusions can be obtained as follows:

The incentive intensity of the government compensation is an increasing function to its supervision over the natural condition. So the government should increase both the observation and supervision intensities and the ecological compensation incentive intensity so as to effectively urge the enterprise's motivation of fulfilling the 'ecological' responsibility.

The endeavor level of the enterprise's fulfilling the 'ecological' responsibility is an increasing function to the government's observation and supervision intensity. That's to say, with the government's observation and supervision intensity of the natural condition increasing, the transparency of the endeavor level of the enterprise's fulfilling the 'ecological' responsibility will rise. And the information asymmetry will be reduced. Therefore, if the government increases the observation and supervision intensity of the natural condition, the enterprise's motivation of fulfilling the 'ecological' responsibility will be promoted and the endeavor level will approach to Pareto optimality.

On the condition that the government observes and supervises the natural condition, the expected output of the enterprise's fulfilling 'ecological' responsibility is much more than the one without the observation and supervision. In other words, the government's observation and supervision of the natural condition is beneficial to the output benefits of the enterprise's fulfilling the 'ecological' responsibility.

\section{Acknowledgments}

This work is supported by the key project of the National Social Science Foundation of China (Proposal No. 11AZD001), Henan Provincial Philosophy Social Science Project of China (Proposal No. 2012CJJ014), Henan Provincial Soft Science Research Project of China (Proposal No. 132400410393) and The Xinxiang University of Science and Technology Innovation Fund Project (Proposal No. 12SB17).

\section{References}

1. Kagan RA, Gunningham N, Thornton D. Explaining corporate environmental performance: how does regulation matter? Law Soc. Rev. 2003;37:51-90.

2. Doonan J, Lanoie P, Laplante B. Determinants of environmental performance in the Canadian pulp and paper industry: an assessment from inside the industry. Ecol. Econ. 2005;55:73-84.

3. Delmas MA, Toffel MW. Organizational responses to environmental demands: opening the black box. Strategic Manage. J. 2008;29:1027-1055.

4. Mirrless J. Notes on welfare economies, information and uncertainty. In: Michael Balch, Daniel McFadden and Shif-yen $\mathrm{Wu}$, eds. Essays on economic behavior under uncertainty. Amsterdam: North-Holland; 1974.

5. Mirrless JA. The optimal structure of authority and incentives within an organization. Bell J. Econ. 1976;7:105-131.

6. Holmstrom B. Moral hazard in teams. Bell J. Econ. 1982;13: 324-340.

7. Theilen B. Simultaneous moral hazard and adverse selection with risk agents. Econ. Lett. 2003;79:283-289. 